\title{
EYES - Energy Efficient Sensor Networks
}

\author{
Paul Havinga ${ }^{1}$, Sandro Etalle ${ }^{1}$, Holger $\mathrm{Karl}^{2}$, Chiara Petrioli ${ }^{3}$, \\ Michele Zorzi ${ }^{4}$, Harry Kip ${ }^{5}$, and Thomas Lentsch ${ }^{6}$ \\ 1 University of Twente, The Netherlands \\ p.j.m.havinga@utwente.nl \\ 2 Technical University of Berlin, Germany \\ 3 Rome University "La Sapienza", Italy \\ ${ }^{4}$ CNIT, Italy \\ 5 Nedap N.V., The Netherlands \\ 6 Infineon Technologies, Austria
}

\begin{abstract}
The EYES project (IST-2001-34734) is a three years European research project on self-organizing and collaborative energy-efficient sensor networks. It will address the convergence of distributed information processing, wireless communications, and mobile computing.

The goal of the project is to develop the architecture and the technology which enables the creation of a new generation of sensors that can effectively network together so as to provide a flexible platform for the support of a large variety of mobile sensor network applications.

This document gives an overview of the EYES project.
\end{abstract}

\section{Introduction}

The vision of ubiquitous computing requires the development of devices and technologies, which can be pervasive without being intrusive. The basic components of such a smart environment will be small nodes with sensing and wireless communications capabilities, able to organize flexibly into a network for data collection and delivery. Realising such a network presents very significant challenges, especially at the architectural and protocol/software level. Major steps forward are required in the field of communications protocols, data processing, and applications support. The EYES project (IST-2001-34734) is a three years European research project on self-organizing and collaborative energy-efficient sensor networks. It will address the convergence of distributed information processing, wireless communications, and mobile computing.

The goal of the project is to develop the architecture and the technology which enables the creation of a new generation of sensors that can effectively network together so as to provide a flexible platform for the support of a large variety of mobile sensor network applications.

\section{$1.1 \quad$ Ubiquitous Computing}

"Ubiquitous Computing", a phrase that the late Mark Weiser (1952 - 1999) described in 1988 as "the calm technology, that recedes into the background of our 
lives", matures from the vision of the Nineties to the reality of the young millennium, enabling increasing mobility and interaction of services and applications in a large variety of areas in daily life. Recently, we have seen rapid progress in developing a new off-the-desktop computing paradigm that moves towards the notion of a pervasive, wearable, unobtrusive, disappearing, or invisible computer. Sensors are tiny devices capable of capturing physical information, such as heat, light or motion, about an environment. Rapid advances in micro-electromechanical systems (MEMS), digital circuitry, and wireless communication have enabled a new generation of tiny, inexpensive, networked sensors. Embedding millions of sensors into an environment creates a digital skin or wireless network of sensors. These massively distributed sensor networks, communicate with one another and summarize the immense amounts of low-level information to produce data representative of the overall environment. From collaboration between (large) groups of sensor nodes, intelligent behaviour can emerge that surpasses the limited capabilities of individual sensor nodes.

Collaborative, smart sensor networks present information in a qualitative, human-interpretable form, which allows people (or computers) to respond intelligently. Sensor networks will change the way we work and live. Sensor network systems will enhance usability of appliances, and provide condition-based maintenance in the home. These devices will enable fundamental changes in applications spanning the home, office, clinic, factory, vehicle, metropolitan area, and the global environment. Sensor node technology enables data collection and processing in a variety of situations, for applications, which include environmental monitoring, context-aware personal assistants (tracking of location, activity, and environment of the user) home security, machine failure diagnosis, medical monitoring, and surveillance and monitoring for security.

Due to the high volume of energy constrained sensors in such networks and their use in possibly harsh environments, replacement of batteries in sensor nodes is not practical in these systems, their lifetime is however required to extend over years. Even though preliminary works have identified some of the requirements for sensor network implementation stressing the role of energy-efficient design, and have proposed preliminary solutions for some of the critical issues such as energy-efficient routing, some important areas such as self-configurable and reconfigurable energy-efficient architectures, maintainability, security, as well as application-dependent issues like reliability, have been at least partially overlooked by the current research. Also an integrated approach to energy-efficient design is lacking and would be extremely beneficial for sensor network design.

\section{Objectives}

We believe that the solution of these problems at their core depends on highly distributed, reliable, and secure information systems that can evolve and adapt to radical changes in their environment, delivering information services that adapt to the people and services that need them. These systems must easily and naturally integrate devices, ranging from tiny sensors and actuators to hand- 
held information appliances. Such devices must be connected by short-range wireless networks as well as by high-bandwidth backbones. Data and services must be secure, reliable, and high-performance, even if part of the system is down, disconnected, under repair, or under attack. The system must configure, install, diagnose, maintain, and improve itself - this applies especially to the vast numbers of sensors that will be cheap, widely dispersed, and even disposable. Finally, it must allow vast quantities of data to be easily and reliably accessed, manipulated, disseminated, and used in a customized fashion by users.

The goal of the EYES project is to develop the architecture and the technology, which enables the creation of a new generation of self-organizing and collaborative sensors that can effectively network together so as to provide a flexible platform for the support of a large variety of mobile sensor network applications.

We will also show the feasibility of the concepts and technologies developed by means of a prototype in which a sizable network of sensors will be built and some example applications demonstrated.

\section{Description of the Work}

The technical work will focus mostly on architectural, protocol and software issues. This area is in fact believed to be the true bottleneck in current sensor networks. The challenges to face in developing new technologies for sensor networks are the need for the nodes to be smart, self-configurable, capable of networking together, and the inherent poverty of resources of the nodes themselves. The main thrust of the work will therefore be directed towards the development of new architectural schemes and communications protocols and algorithms at multiple layers, taking into account those specific features. In particular, schemes, which are able to work efficiently in the presence of limited energy, processing power and memory, will be developed.

\section{Architecture}

In our approach we define two distinct key system layers of abstraction: the sensor and networking layer, and the distributed services layer. Each layer provides services that may be spontaneously specified and reconfigured.

- The sensor and networking layer contains the sensor nodes (the physical sensor and wireless transmission modules) and the network protocols. Ad-hoc routing protocols allow messages to be forwarded through multiple sensor nodes taking into account the mobility of nodes, and the dynamic change of topology. Communication protocols must be energy-efficient since sensor nodes have very limited energy supply. To provide more efficient dissemination of data, some sensors may process data streams, and provide replication and caching. 
- The distributed services layer contains distributed services for supporting mobile sensor applications. Distributed services co-ordinate with each other to perform decentralized services. These distributed servers may be replicated for higher availability, efficiency and robustness. We have identified two major services. The lookup service supports mobility, instantiation, and reconfiguration. The information service deals with aspects of collecting data. This service allows vast quantities of data to be easily and reliably accessed, manipulated, disseminated, and used in a customized fashion by applications.

On top of this architecture applications can be built using the sensor network and distributed services.

\section{Milestones and Expected Result}

Both theoretical research results and the implementation of a prototype will be produced. Specifically, we will:

- Create new technologies for communications, networking and service provision which take explicitly into account the constraints of sensor networks

- Propose and test a new architecture for flexible support of mobile sensor network applications

- Test ideas by building a prototype sensor network in which to implement the proposed schemes thereby verifying their effectiveness.

\section{$6 \quad$ Project Details}

- Project Reference: IST-2001-34734

- Start Date: 2002-03-01

- End Date: 2005-02-28

- Project budget: 4.730 M-Euro

- Project URL: http://eyes.eu.org/

\section{Participants}

- Universiteit Twente, CTIT, The Netherlands

- Infineon Technologies, Austria

- Consorzio Nazionale Interuniversitario per le Telecomunicazioni, Italy

- N.V. Nederlandsche Apparatenfabriek "Nedap", The Netherlands

- Universita degli Studi di Roma "La Sapienza", Italy

- Technische Universitat Berlin, Germany 